\title{
Thermodynamics, entropy, and stability of thin shells in $2+1$ flat spacetimes
}

\author{
José P. S. Lemoł* and Gonçalo M. Quintd \\ Centro Multidisciplinar de Astrofísica, CENTRA, Departamento de Física, \\ Instituto Superior Técnico - IST, Universidade de Lisboa - UL, \\ Avenida Rovisco Pais 1, 1049-001 Lisboa, Portugal
}

\begin{abstract}
The thermodynamic equilibrium states of a static thin ring shell in a $(2+1)$-dimensional flat spacetime is analyzed. Inside the ring the spacetime is flat, whereas outside it is conical flat. The first law of thermodynamics applied to the thin shell leads to a shell's entropy which is a function of its mass alone. Two simple forms for this mass function are given leading to two different expressions for the entropy. The equations of thermodynamic stability are analyzed resulting in certain allowed regions for the free parameters. Contrary to the usual $(3+1)$-dimensional case this shell's entropy is purely classic, as the only fundamental constant that enters into the problem is the $(2+1)$-dimensional gravitational constant $G_{3}$, which has units of inverse mass.
\end{abstract}

PACS numbers: 04.40.-b, 04.70.Dy

\section{INTRODUCTION}

The study of thermodynamics of gravitational systems has proved important in many respects, such as the understanding that these systems can have negative specific heat, and that the gravitational object par excellence, the black hole, has definite temperature and entropy associated with it (see, e.g., [1]), which in turn indicates that a quantum theory of gravity might be in sight. In particular, a special kind of gravitating system, a thin shell with its surrounding spacetime, is prone to a direct attack of its thermodynamic properties. Indeed, Davies, Ford and Page [2] and Hiscock 3], have shown the usefulness of studying thin shells in $(3+1)$-dimensional general relativistic spacetimes from a thermodynamic viewpoint. Further progress was achieved by Martinez [4], in which several thermodynamic quantities are discussed and a stability analysis of thin shells is performed using the formalism set in [5]. For related studies of thermodynamics of gravitating matter, especially on the verge of becoming a black hole, see [6, 7].

Although (3+1)-dimensional spacetimes are of the greatest interest, it is also pertinent to study thin shells and their properties in $(2+1)$-dimensional spacetimes. The relevance in the study of three-dimensional gravity started with the work of Deser, Jackiw, and 't Hooft [8], where it was shown that, though the corresponding vacuum solution is trivial since it consists of Minkowski spacetime, a point particle distorts it into a conical space with the particle being located at the vertex of the cone, and moreover, moving point particles display nontrivial dynamics. The next simplest object, beyond a point a particle in $2+1$ dimensions, is a thin shell, i.e, a ring dividing two vacua regions, the interior and exterior to the ring itself. A detailed analysis of the dynamics of such a ring, collapsing or expanding was performed in [9], where the usual junction condition formalism [10] was used.

To further understand the thermodynamic properties of gravitational systems we propose to study the thermodynamics of a static thin ring shell in $(2+1)$-dimensional

\footnotetext{
*Electronic address: joselemos@ist.utl.pt
}

${ }^{\dagger}$ Electronic address: goncalo.quinta@ist.utl.pt general relativity. By making use of the appropriate junction conditions for general relativity [10], one can determine the pressure and rest mass of the shell in order for it to be static with interior and exterior spacetimes both flat. Using then the formalism developed by Martinez [4], with the thermodynamic theory as presented in [5], one can find generic expressions for the shell's entropy, which upon some minimal assumptions about the structure of the matter fields making the system, i.e., an ansatz for the shell temperature in terms of the gravitational quantities that characterize the system, yields a definite expression for the entropy of the shell, and permits a stability analysis.

The paper is organized as follows. In Sec. III, we will compute the components of the extrinsic curvature of the shell that will lead to the shell's linear density and pressure. In Sec. III we will use these results by directly inserting them in the first law of thermodynamics to obtain the differential of the entropy, which will naturally have a degree of freedom parametrized by an arbitrary function associated with the matter fields that make up the shell. Two phenomenological expressions will be considered for the arbitrary function, namely a simple power law of the rest mass and a power law of a quadratic expression of the rest mass, which will lead to two different expressions for the entropy. In Sec. IV we will analyze the thermodynamic stability of the system for each entropy function by calculating the allowed intervals of the free parameters in order for the shell to remain thermodynamically stable. In Sec. V we conclude.

\section{THE THIN SHELL SPACETIME}

In $2+1$ dimensions Einstein's equation takes the form

$$
G_{a b}=8 \pi G_{3} T_{a b},
$$

where $G_{a b}$ is the (2+1)-dimensional Einstein tensor, $T_{a b}$ is the stress-energy tensor, and $G_{3}$ is the gravitational constant in $2+1$ dimensions. $G_{3}$ has units of inverse mass. The speed of light is taken to be equal to 1 .

To find the solution for a thin shell in a $(2+1)$ dimensional spacetime, we follow [10] and start by considering a one-dimensional timelike hypersurface $\Sigma$ that 
partitions spacetime into two spherically symmetric regions: an inner region $\mathcal{V}_{-}$and an outer region $\mathcal{V}_{+}$.

Inside the hypersurface we will use flat-polar coordinates $(t, r, \theta)$ for a flat metric with line element

$$
d s^{2}=-d t^{2}+d r^{2}+r^{2} d \theta^{2}, \quad r<R,
$$

where $r=R$ is the radius of the thin-shell hypersurface.

On the outside of the shell, the spacetime is again flat but the presence of matter justifies the use of conicalpolar coordinates $(t, r, \theta)$, allowing the line element to be written as $d s^{2}=-\beta^{2} d t^{2}+d r^{2}+r^{2} \alpha^{2} d \theta^{2}$, for $r>R$, and some constant $\alpha$. It is preferable to make the change $\alpha r \rightarrow r$, and without loss of generality one can put $\beta=$ $\alpha$, so that the metric takes the form

$$
d s^{2}=-\alpha^{2} d t^{2}+\frac{d r^{2}}{\alpha^{2}}+r^{2} d \theta^{2}, \quad r>R .
$$

The metric (3) has a conical singularity at $r=0$ if the thin-shell hypersurface has a radius $R(\tau) \rightarrow 0$, i.e., it turns into a point particle.

The parametric equations of the thin shell hypersurface $\Sigma$ are described by $r=R(\tau), t=T(\tau)$, where $\tau$ is the proper time on the thin shell hypersurface. Choosing coordinates $(\tau, \theta)$, we have an induced metric $h_{a b}$ given by

$$
d s_{\Sigma}^{2}=-d \tau^{2}+R^{2}(\tau) d \theta^{2} .
$$

The first junction condition states that in order for $\mathcal{V}_{+}$ and $\mathcal{V}_{-}$to be joined smoothly at $\Sigma$, the induced metric seen from both sides must satisfy $\left[h_{a b}\right]=0$, where the parentheses symbolize the jump in the metric across the hypersurface. This condition leads to the relations

$$
\alpha^{2} \dot{T}^{2}-\frac{\dot{R}^{2}}{\alpha^{2}}=\dot{T}^{2}-\dot{R}^{2}=1
$$

where the dot denotes differentiation with respect to $\tau$.

The second junction condition involves the extrinsic curvature $K^{a}{ }_{b}$ defined as

$$
K^{a}{ }_{b}=h^{a c} h_{b}^{d} \nabla_{c} n_{d}
$$

where $\nabla_{c}$ denotes covariant derivation. In general, when there is a thin matter shell, the boundary stress-energy tensor $S^{a}{ }_{b}$ is related to the jump in extrinsic curvature by

$$
S_{b}^{a}=-\frac{1}{8 \pi G_{3}}\left(\left[K_{b}^{a}\right]-[K] h_{b}^{a}\right)
$$

where $K=h^{b}{ }_{a} K^{a}{ }_{b}$ and $G_{3}$ is the gravitational constant in $2+1$ dimensions. From the line elements given in Eqs. (2) and (3) and using Eq. (5), one can compute the nonzero components of $K^{a}{ }_{b}$,

$$
\begin{aligned}
& K_{+\tau}^{\tau}=\frac{\ddot{R}}{\sqrt{\alpha^{2}+\dot{R}^{2}}}, \\
& K_{-\tau}^{\tau}=\frac{\ddot{R}}{\sqrt{1+\dot{R}^{2}}}, \\
& K_{+\theta}^{\theta}=\frac{1}{R} \sqrt{\alpha^{2}+\dot{R}^{2}}, \\
& K_{-\theta}^{\theta}=\frac{1}{R} \sqrt{1+\dot{R}^{2}},
\end{aligned}
$$

which, in turn, lead to the nonzero components of the linear boundary stress-energy tensor

$$
\begin{aligned}
S^{\tau}{ }_{\tau} & =\frac{1}{8 \pi G_{3}} \frac{\sqrt{\alpha^{2}+\dot{R}^{2}}-\sqrt{1+\dot{R}^{2}}}{R} \\
S^{\theta}{ }_{\theta} & =\frac{1}{8 \pi G_{3}}\left(\frac{\ddot{R}}{\sqrt{\alpha^{2}+\dot{R}^{2}}}-\frac{\ddot{R}}{\sqrt{1+\dot{R}^{2}}}\right) .
\end{aligned}
$$

Assuming the shell is a perfect fluid, the specific physical form of the linear boundary tensor is

$$
S^{a}{ }_{b}=(\lambda+p) u^{a} u_{b}+p h_{b}^{a},
$$

where $p$ is the linear pressure and $\lambda$ is the linear mass density of the shell. So for a perfect fluid $S^{\tau}{ }_{\tau}=-\lambda$ and $S^{\theta}{ }_{\theta}=p$. Thus, from Eqs. (12) and (13), it follows that

$$
\begin{aligned}
& \lambda=\frac{1}{8 \pi G_{3}} \frac{\sqrt{1+\dot{R}^{2}}-\sqrt{\alpha^{2}+\dot{R}^{2}}}{R} \\
& p=\frac{1}{8 \pi G_{3}}\left(\frac{\ddot{R}}{\sqrt{\alpha^{2}+\dot{R}^{2}}}-\frac{\ddot{R}}{\sqrt{1+\dot{R}^{2}}}\right)
\end{aligned}
$$

This set of equations has been previously found and studied in [9] in a $2+1$ dynamical shell collapsing setting. Taking the static limit $\dot{R}=\ddot{R}=0$ in the above equations and using the definition of the shell's rest mass

$$
M=2 \pi R \lambda,
$$

we have immediately

$$
\begin{aligned}
M & =\frac{1-\alpha}{4 G_{3}} \\
p & =0 .
\end{aligned}
$$

To have a properly defined shell radius one has to impose $\alpha>0$. Imposing positive mass it follows $0<\alpha<1$, whereas negative masses appear in the range $1<\alpha<$ $\infty$. From Eq. (19) we see that in order for the shell to be static in a $(2+1)$-dimensional spacetime, its linear pressure must vanish.

We now want to study this static thin shell spacetime 9] from a thermodynamics point of view. Through the formalism presented in [1] and developed by Martinez [4] for the thermodynamics of thin shells, we study the thermodynamics and find the entropy of these thin shells in $(2+1)$ flat dimensions.

\section{THERMODYNAMICS, ENTROPY EQUATION FOR THE SHELL, AND STABILITY}

We assume that the entropy of the shell can be expressed in terms of its characteristics, namely, its rest mass $M$ and radius $R$. It is more useful, though equivalent, to work with $M$ and $A[4]$, where $A$ is the area (here circumference) of the shell, $A \equiv 2 \pi R$. Thus we write,

$$
S=S(M, A) .
$$

Assuming further that the shell is at some local temperature $T$, an explicit expression for $S$ can then be found by directly integrating the first law of thermodynamics

$$
T d S=d M+p d A .
$$


By putting $p=0$ as given in Eq. (19), the first law for the $(2+1)$-dimensional thin shell spacetime simplifies to

$$
T d S=d M .
$$

Equation (22) can be integrated provided that the integrability conditions are satisfied. In this case there is only one condition. It states that $T=T(M)$, i.e., the temperature $T$ is a function of the mass $M$ alone. This simple dependence stems from the fact that $p=0$ as given in Eq. (19). Then, the most general expression for $S$ is

$$
S(M)=\int \beta(M) d M+S_{0}
$$

where $\beta \equiv 1 / T$ is the local inverse temperature of the shell at the equilibrium position $r=R$, and $S_{0}$ is an integration constant. Note that $\beta(M)$ is an arbitrary function of the mass that can be specified once the specific matter fields that constitute the shell are known.

Local intrinsic stability of the shell can also be studied. The former is guaranteed as long as all the following inequalities are verified

$$
\begin{gathered}
\left(\frac{\partial^{2} S}{\partial M^{2}}\right)_{A} \leq 0 \\
\left(\frac{\partial^{2} S}{\partial A^{2}}\right)_{M} \leq 0 \\
\left(\frac{\partial^{2} S}{\partial M^{2}}\right)\left(\frac{\partial^{2} S}{\partial A^{2}}\right)-\left(\frac{\partial^{2} S}{\partial M \partial A}\right)^{2} \geq 0,
\end{gathered}
$$

where the formalism developed in [5] is being followed.

\section{TWO SPECIFIC EQUATIONS OF STATE FOR THE THIN SHELL MATTER: ENTROPY AND STABILITY}

\section{A. The simplest equation of state}

The simplest form that can be considered for $\beta(M)$ is a power law,

$$
\beta(M)=\gamma G_{3}^{(1+u)} M^{u}
$$

where we are assuming $M \geq 0, \gamma$ and $u$ are free parameters with $\gamma>0$ to guarantee positive temperature, the factor $G_{3}$ must be present for dimensional reasons, and Boltzmann's constant is set to 1 . In $3+1$ dimensions, Planck's length $l_{\mathrm{p}}$, with $l_{\mathrm{p}}=\sqrt{G_{4} h}\left(G_{4}\right.$ being the gravitational constant in four-dimensional spacetime and $h$ Planck's constant), appears naturally in the temperature of a thin shell, since for a given mass there is always an intrinsic length associated with it (the gravitational radius of the system), and so to have the correct units for the entropy one must resort to $l_{\mathrm{p}}$. However, here in flat $2+1$ dimensions there is no intrinsic spacetime radius, and so Planck's length does not appear in this analysis at all. This problem is thus purely classic and $G_{3}$, with units of inverse mass, suffices to set the scale.

When $\beta(M)$ has the form given in Eq. (27), one can substitute this in (23) to get

$$
S(M)=\frac{\gamma}{u+1}\left(G_{3} M\right)^{(1+u)}+S_{0}, \quad \text { for } u \neq-1,
$$

and

$$
S(M)=\gamma \ln \left(G_{3} M\right)+S_{0}, \quad \text { for } u=-1 .
$$

Although the values of the parameters $\gamma$ and $u$ cannot be calculated without first specifying the nature of the matter fields, it is possible to constrain them such that physical equilibrium states of the shell are possible. Starting with $S_{0}$, it is natural to assume that a zero mass shell should have zero entropy, i.e., $S(M \rightarrow 0)=$ $\int \beta(M) d M+S_{0} \rightarrow 0$. It is seen directly from Eq. (27) that the entropy diverges when $M \rightarrow 0$ for $u \leq-1$. Therefore the above normalization condition can only be satisfied for $u>-1$ and $S_{0}=0$.

In relation to stability, it is seen that conditions (25) and (26) are automatically satisfied for any $u$. On the other hand, one can find that condition (24) can only be satisfied provided that $u \leq 0$. Thus we conclude that assuming a power law equation of the form (27), stability of the shell is possible for any $M \geq 0$ as long as the parameter values of $u$ are restricted to

$$
-1<u \leq 0 .
$$

One can also consider negative values of $M$, i.e., $\alpha>1$. The relation (27) would be of the same form with the proviso that one takes the absolute values of $M$. The same results would follow.

\section{B. A more contrived equation of state}

Another possibility for $\beta(M)$ could be a quadratic function in $M$, of the form $\beta(M)=\delta G_{3}^{(1+a)}\left(M+C M^{2}\right)^{a}$, where $\delta$ and $a$ are some parameters with $\delta>0$ to guarantee positive temperature, and $C$ is some constant. The constant $C$, however, has a natural connection to Eq. (18). Indeed, defining $m \equiv M+C M^{2}$ we can solve for $M$, obtaining the physical solution $M=\frac{-1+\sqrt{1+4 C m}}{2 C}$. Comparing with Eq. (18), we can make the association $C=-2 G_{3}$ and so $\alpha=\sqrt{1+4 C m}=\sqrt{1-8 G_{3} m}$, thus arriving at the natural quadratic expression for $\beta$

$$
\beta(M)=\delta G_{3}^{(1+a)}\left(M-2 G_{3} M^{2}\right)^{a} .
$$

Equation (31) is most easily integrated in the variable $m$. Changing from $M$ to $m$ in Eq. (23), defining the parameter $\eta=\delta / \alpha$, and changing back again to $M$, we obtain the entropy

$$
\begin{array}{r}
S(M)=\frac{\eta}{a+1} G_{3}^{(1+a)}\left(M-2 G_{3} M^{2}\right)^{(1+a)}+S_{0}, \\
\text { for } a \neq-1
\end{array}
$$

and

$$
S(M)=\eta \ln \left[G_{3}\left(M-2 G_{3} M^{2}\right)\right]+S_{0}, \quad \text { for } a=-1 .
$$


Again, although the values of the parameters $\eta$ and a cannot be calculated without first specifying the nature of the matter fields, it is possible to constrain them such that physical equilibrium states of the shell are possible. Starting with $S_{0}$, it is natural to assume that a zero mass shell should have zero entropy, i.e., $S(M \rightarrow 0)=\int \beta(M) d M+S_{0} \rightarrow 0$. It is seen directly from (31) that the entropy diverges when $M \rightarrow 0$ for $a>-1$. Therefore the above normalization condition can only be satisfied for $a>-1$ and $S_{0}=0$.

From Eq. (31), the stability equations (25) and (26) are automatically satisfied since the entropy does not depend on $A$. Also, as mentioned above, $\alpha>0$ to have a physical acceptable solution. Considering Eq. (24), it is possible to show that it implies the inequality

$$
(2 a+1) \alpha^{2}-1 \leq 0 .
$$

We can study the two cases, $M>0$ and $M<0$.

For $M>0, \alpha$ is in the range $0<\alpha \leq 1$. In this case Eq. (34) is automatically satisfied if the exponent $a$ obeys $a \leq 0$. It is also satisfied for $a>0$ but only if $0<\alpha \leq \sqrt{\frac{1}{2 a+1}}$. Equivalently, this means that the rest mass $M$ of the shell must be within the range

$$
\frac{1-\sqrt{1-2 a /(2 a+1)}}{4 G_{3}}<M \leq \frac{1}{4 G_{3}},
$$

for $a>0$.

For $M<0$, we know that $\alpha>1$, and Eq. (34) requires additionally that $\alpha \leq \sqrt{\frac{1}{2 a+1}}$. In terms of rest mass $M$, this represents the range

$$
\frac{1-\sqrt{1-2 a /(2 a+1)}}{4 G_{3}}<M<0 .
$$

However, since $\sqrt{\frac{1}{2 a+1}}>1$ and $2 a+1>0$, we see that the analysis for $M<0$ is only valid for parameter values $-1 / 2<a<0$.

\section{CONCLUSIONS}

General relativity in a $(2+1)$-dimensional spacetime has no curvature in empty space but in matter distributions curvature may still exist. Einstein's equation thus still plays a role in determining the required pressure and energy of a static thin shell (or ring in this $(2+1)$ dimensional setting). Indeed, we have seen that in this situation the pressure must be zero and the rest mass of the shell must satisfy Eq. (18).

Upon using the first law of thermodynamics we have found a specific differential equation for the entropy of the ring that contained a degree of freedom encoded in the inverse temperature $\beta$. We have chosen the two simple ansatz for the inverse temperature, a power law on the shell's rest mass and a quadratic form of it, obtaining two distinct expressions for the ring's entropy. This shell's entropy is purely classic, as the only fundamental constant that enters into the problem is the $(2+1)$ dimensional gravitational constant $G_{3}$, which has units of inverse mass. This entropy could perhaps be explained quantically if the shell's mass is given in terms of the mass of its elementary constituents and a fundamental theory for the mass of those constituents particles is at hand. A thermodynamic stability analysis yielded the range for the allowed parameters, revealing that the shell's rest mass must be confined to a given interval if the shell is to be stable.

Our results are of importance if one uses a concrete model for the shell, e.g., a model involving fundamental scalar fields. One would then extract from the model the specific equation of state with its precise values for the exponents and constants, and could immediately determine through the above equations whether it would be thermodynamic stable.

We also note that these ring shell spacetimes when extended into $3+1$ dimensions, through the use of a trivial coordinate $z$ say, represent infinite cylinders. Thus this thermodynamic study also holds in $3+1$ general relativity for those cylindrical thin shells.

\section{Acknowledgments}

We thank FCT-Portugal for financial support through Projects No. PTDC/FIS/098962/2008 and No. PEstOE/FIS/UI0099/2011.
[1] H. Braden, J. D. Brown, B. F. Whiting, and J. W. York, Phys. Rev. D 42, 3376 (1990).

[2] P. C. W. Davies, L. H. Ford, and D. N. Page, Phys. Rev. D 34, 1700 (1986).

[3] W. A. Hiscock, Phys. Rev. D 53, 7062 (1996).

[4] E. A. Martinez, Phys. Rev. D 40, 1336 (1989).

[5] H. B. Callen, Thermodynamics and an Introduction to Thermostatistics (Wiley, New York, 1985).

[6] J. P. S. Lemos and O. B. Zaslavskii, Phys. Rev. D 81,
$064012(2010)$.

[7] J. P. S. Lemos and O. B. Zaslavskii, Phys. Lett. B 695, 37 (2011)

[8] S. Deser, R. Jackiw, and G. 't Hooft, Ann. Phys. (N.Y.) 152, 220 (1984).

[9] R. B. Mann and J. J. Oh, Phys. Rev. D 74, 124016 (2006).

[10] W. Israel, Nuovo Cimento B 44, 1 (1966). 